\title{
Generating a network reliability formula by using binary decision diagrams
}

\author{
Yasuhiro Ikeda ${ }^{\text {a) }}$, Ryoichi Kawahara, and Hiroshi Saito \\ NTT Network Technology Laboratories, NTT Corporation, \\ 3-9-11 Midori-cho, Musashino-shi, Tokyo 180-8585, Japan \\ a)ikeda.yasuhiro@lab.ntt.co.jp
}

\begin{abstract}
Evaluating network reliability is essential for network management. In the case that the failure probabilities of nodes and links change in an evaluation, iterative calculation of the reliability is required. In large networks, however, this calculation takes considerable time; thus, iterative calculation cannot be done within a practical amount of time. In this paper, we present a method for generating network reliability formulas that is based on the reliability calculation method with binary decision diagrams (BDD). We show that the time taken to calculate reliability can be reduced by the formula expression in some orders of magnitude compared with the conventional BDD calculation method, which excludes the overhead of formula generation.
\end{abstract}

Keywords: reliability, formulation, BDD

Classification: Fundamental Theories for Communications

\section{References}

[1] J. Abraham, "An improved algorithm for network reliability," IEEE Trans. Reliab., vol. 28, no. 1, pp. 58-61, 1979. DOI:10.1109/TR.1979.5220476

[2] Y. Yoo and N. Deo, "A comparison of algorithms for terminal-pair reliability," IEEE Trans. Reliab., vol. 37, no. 2, pp. 210-215, 1988. DOI:10.1109/24.3743

[3] S.-Y. Kuo, F.-M. Yeh, and H.-Y. Lin, "Efficient and exact reliability evaluation for networks with imperfect vertices," IEEE Trans. Reliab., vol. 56, no. 2, pp. 288-300, 2007. DOI:10.1109/TR.2007.896770

[4] "Sympy [Online]," http://www.sympy.org/en/index.html.

\section{Introduction}

Reliability, which is the probability that the facilities in a network can communicate with each other, is a fundamental performance measure in communication networks. In network management, a network is often expressed by a graph. Facilities, such as routers and servers, are expressed as nodes, and cables and fibers are expressed as links in the graph. The probability that facilities communicate normally is considered in the graph as the probability that corresponding nodes and links have a "success" status, where the nodes and links do not fail in connection. 
We call this probability the "success probability." The reliability among $k$ facilities such as servers is calculated as the probability that any set of corresponding nodes can establish a path with nodes and links that have a success status. There have been several methods proposed for calculating network reliability $[1,2,3]$. Methods that use the binary decision diagram (BDD) are very efficient [3].

Network reliability is evaluated in network planning. For example, we can examine node-pair reliability, that is, reliability with $k=2$, for all node pairs in a candidate network topology by giving success probabilities to all nodes and links, and find which node pair is likely disconnected. In the case that the type of nodes and links should also be determined, we should iteratively evaluate the reliability for each node pair by giving the success probabilities corresponding to the candidate type of nodes and links. In large networks, however, calculating reliability requires considerable time since the conventional method needs to consider success and failure cases for each node and link with a given success probability for each. Calculation for a node pair is required for each pattern of success probabilities; thus, calculation for all the node pairs cannot be done in a practical amount of time.

However, reliability should become a function of success probability. If a formula that expresses the network reliability of a node pair is derived once, we can calculate the reliability only by substituting the success probability with the obtained formula, not by considering success or failure for all nodes and links.

In this paper, we propose a method for generating a network reliability formula by using BDD. Section 2 explains BDD, and the generation method is discussed in Section 3. Section 4 shows the simulation results, and we conclude the paper in Section 5.

\section{Reliability calculation with BDD}

In this section, we give a brief summary of BDD. Here, we assume $k=2$ and only consider link failure for simplicity. However, the method can be generalized.

In the network in Fig. 1(a), the Boolean function of the set of required links for connection between $s$ and $t$ is

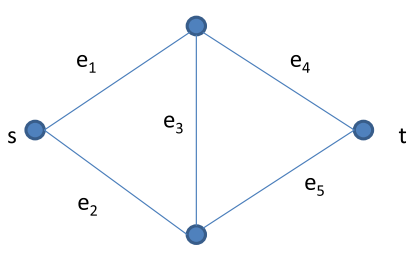

(a) Network

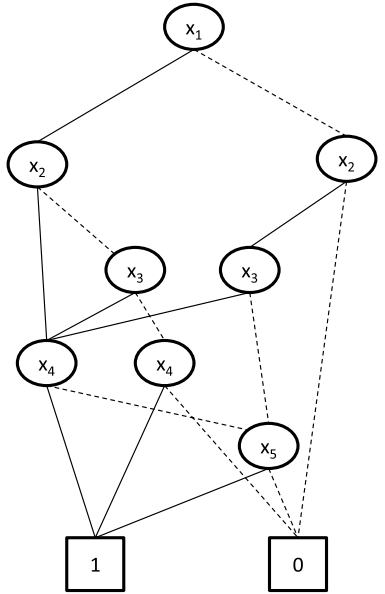

(b) BDD 


$$
x_{1} \cap\left(x_{3} \cap x_{5} \cup x_{4}\right) \cup x_{2} \cap\left(x_{5} \cup x_{3} \cap x_{4}\right),
$$

where $x_{i}$ is a Boolean variable that is 1 if $e_{i}$ is a success link and 0 if not. The Boolean function for connectivity between $s$ and $t$ in network $G$ can be derived by the recursive procedure [3]. By encoding AND and OR in the procedure by BDD operation [3], the Boolean function can be expressed by a BDD graph (see Fig. 1(b)). In the graph, the edges are directed from top to bottom. The solid and dashed edges from vertice $x_{i}$ express $x_{i}=1$ and $x_{i}=0$, respectively. The terminal vertices 1 and 0 express if the boolean function is TRUE(1) or FALSE(0), respectively, with the set of $x_{i}$, which leads to the vertices.

With BDD graph $f$, the reliability can be calculated as

$$
\operatorname{Prob}(f)=P(\tilde{x}) \cdot \operatorname{Prob}\left(f_{\tilde{x}=1}\right)+(1-P(\tilde{x})) \cdot \operatorname{Prob}\left(f_{\tilde{x}=0}\right),
$$

where $\tilde{x}$ is the variable of the top vertice in $f, P(\tilde{x})$ is the success probability of the corresponding link, and $f_{\tilde{x}=1}\left(f_{\tilde{x}=0}\right)$ is the BDD graph connected to the solid (dashed) edge from vertice $\tilde{x}$. $\operatorname{Prob}(f)$ is $1(0)$ if $f$ is the terminal vertice $1(0)$. $\operatorname{Prob}(f)$ calculates the reliability by considering the combination of success $[P(\tilde{x})]$ and failure $[1-P(\tilde{x})]$ patterns for each link, where redundant calculations are eliminated [3]. In addition, overlapping subgraphs are collected in one subgraph in BDD, and a hash table can be used to reduce the calculation [3]. Calculating reliability with BDD, however, still requires considerable recursive calls and takes more time as the size of a BDD graph grows because of network largeness or complexity.

\section{Formula generation with BDD}

The calculation of reliability with BDD is executed with the success probability $P(\tilde{x})$ for each link, and the value is output. Since the BDD calculation method requires only simple multiplication and summation between the probabilities and the redundant calculations are omitted, we can efficiently obtain a formula expression of the reliability by giving the success probabilities as symbols, not as values, and outputting the character string that represents the arithmetic operation between the symbols.

Algorithm 1 shows the procedure. Here, quotation marks are used around words to express that the words are returned as the character string. With BDD in Fig. 1(b), for example, $\operatorname{REL}(f)$ outputs the following formula. $p_{1} \times\left(p_{2} \times\left(p_{4}+\right.\right.$ $\left.\left.\left(1-p_{4}\right) \times p_{5}\right)+\left(1-p_{2}\right) \times\left(p_{3} \times\left(p_{4}+\left(1-p_{4}\right) \times p_{5}\right)+\left(1-p_{3}\right) \times p_{4}\right)\right)+(1-$ $\left.p_{1}\right) \times\left(p_{2} \times\left(p_{3} \times\left(p_{4}+\left(1-p_{4}\right) \times p_{5}\right)+\left(1-p_{3}\right) \times p_{5}\right)\right)$, which represents the network reliability formula.

With the tools for symbolic mathematics, SymPy [4] for example, the output formula can be simplified. By using simplification method expand() in SymPy, the formula previously noted is simplified as $2 p_{1} p_{2} p_{3} p_{4} p_{5}-p_{1} p_{2} p_{3} p_{4}-p_{1} p_{2} p_{3} p_{5}-$ $p_{1} p_{2} p_{4} p_{5}-p_{1} p_{3} p_{4} p_{5}+p_{1} p_{3} p_{5}+p_{1} p_{4}-p_{2} p_{3} p_{4} p_{5}+p_{2} p_{3} p_{4}+p_{2} p_{5}$, where the multiplication symbols are omitted. 


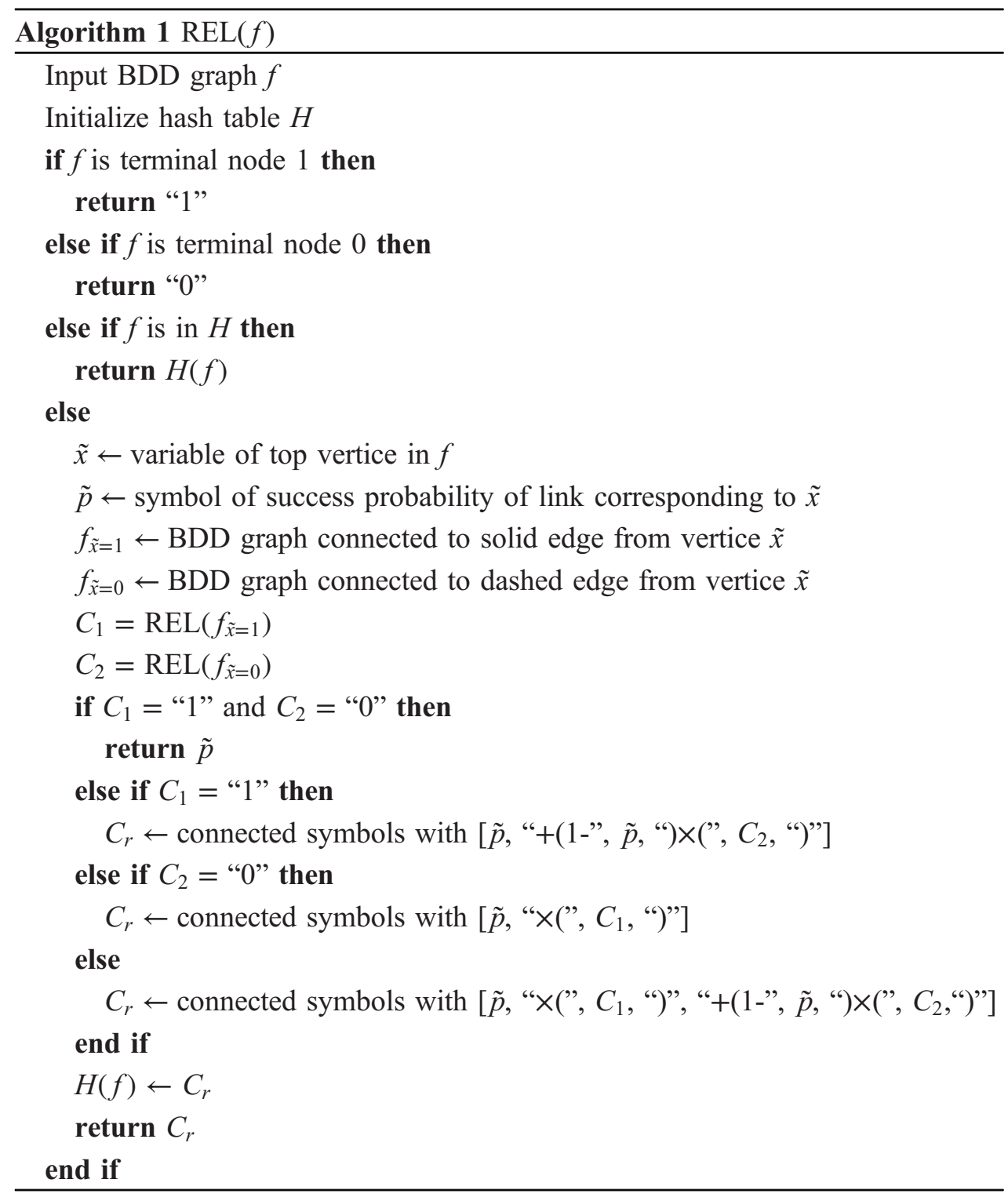

When most of the success probability is given as a value and we want to express only the rest as symbols, the formula can be simplified further with the tools by substituting the known values with the corresponding symbols. For example, by giving $p_{2}, p_{3}, p_{4}$, and $p_{5}$ as 0.9 and assuming $p_{1}$ as a variable, the formula previously noted becomes $0.1062 p_{1}+0.8829$.

\section{Simulation results}

We compared the calculation times between the conventional BDD method and proposed method. For the evaluation, we used a $3 \times 3$ grid network (see Fig. 2).

Table I shows the evaluation results of the computation time, where "gen." is the time taken to generate the formula and "cal." is the time taken to calculate the reliability for all node pairs. For one node pair, the reliability is calculated once with the given success probabilities. BDD (conv.) is the conventional BDD method. formula (naive) is the formulation without any simplification, formula (simplified) is that with simplification of the whole formula, and formula (partial) is the formulation where the success probabilities of $e_{1}, e_{3}, e_{7}$, and $e_{9}$ are given as the 
Table I. Evaluation results of computation time (sec)

\begin{tabular}{|c|c|c|c|c|}
\hline & $B D D$ (conv.) & formula (naive) & formula (simplified) & formula (partial) \\
\hline gen. & - & $1.56 \times 10^{-2}$ & $1.01 \times 10$ & 1.35 \\
\hline cal. & $1.36 \times 10^{-2}$ & $3.09 \times 10^{-3}$ & $1.16 \times 10^{-3}$ & $1.61 \times 10^{-4}$ \\
\hline
\end{tabular}

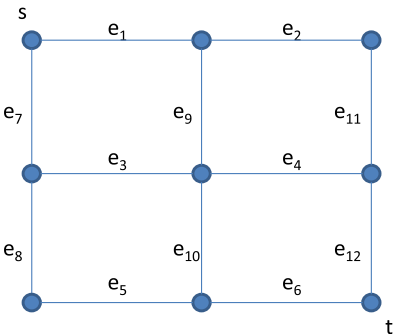

Fig. 2. Network for evaluation

symbolic variables and those of the rest as values. The computation was executed on Python 2.7.6 on CentOS 6.6 with $6 \mathrm{~GB}$ of memory $\times 6$ and a $1.9-\mathrm{GHz}$ $\mathrm{CPU} \times 2$. In formula (simplified) and formula (partial), the simplification of the formula was processed by SymPy [4]. Although the generation of the formula in formula (naive) requires the same time as the calculation in $B D D$ (conv.) and that in formula (simplified) and formula (partial) take more time due to the simplification procedure, the time taken to calculate reliability was one to two orders of magnitude lower. Since generating a formula is needed only once, calculation with the formula is effective when the iterative calculation of the reliability is required.

We also compared the calculation time of the reliability between $B D D$ (conv.) and formula (partial), where the network was a $5 \times 5$ grid and the success probabilities of seven links in the left top of the grid were only given as symbolic objects in formula (partial). The calculation time in $B D D$ (conv.) for all node pairs was $1.46 \times 10(\mathrm{sec})$. In comparison, the generation time of the formula and the calculation time for all node pairs in formula (partial) were $1.13 \times 10^{4}(\mathrm{sec})$ and $6.14 \times 10^{-4}(\mathrm{sec})$, respectively. If the number of the types of the link is four, the combinations of the types of seven links is $4^{7}=16,384$. Thus, the total time needed to evaluate the reliabilities is about 66 (hours) in $B D D$ (conv.), whereas that in formula (partial) is about 3 (hours). As the number of the combinations of the types increases, formula (partial) becomes more efficient since the calculation time in formula (partial) is negligible.

\section{Conclusion}

We proposed a method for generating a network reliability formula by using BDD. Although generating a formula requires overhead, the calculation time of the reliability can be reduced some orders of magnitude compared with the conventional BDD method. 